\title{
COVERING INTERSECTING BI-SET FAMILIES UNDER MATROID CONSTRAINTS*
}

\author{
KRISTÓF BÉRCZI ${ }^{\dagger}$, TAMÁS KIRÁLY ${ }^{\dagger}$, AND YUSUKE KOBAYASHI ${ }^{\ddagger}$
}

\begin{abstract}
Edmonds's fundamental theorem on arborescences in [J. Edmonds, Edge-disjoint branchings, in Combinatorial Algorithms, Courant Comput. Sci. Sympos. 9, Algorithmics Press, New York, 1973, pp. 91-96] characterizes the existence of $k$ pairwise arc-disjoint spanning arborescences with the same root in a directed graph. In [L. Lovász, J. Combinatorial Theory Ser. B, 21 (1976), pp. 96-103], Lovász gave an elegant alternative proof which became the basis of many extensions of Edmonds's result. In this paper, we use a modification of Lovász's method to prove a theorem on covering intersecting bi-set families under matroid constraints. Our result can be considered as an extension of previous results on packing arborescences. We also investigate the algorithmic aspects of the problem and present a polynomial-time algorithm for solving the corresponding optimization problem.
\end{abstract}

Key words. packing arborescences, bi-sets, intersecting families, matroids

AMS subject classifications. 05C70, 05C 85

DOI. $10.1137 / 15 \mathrm{M} 1049099$

1. Introduction. Let $D=(V, A)$ be a directed graph (or digraph, for short). For disjoint sets $X, Y \subseteq V$, we say that $Y$ is reachable from $X$ if there is a directed path from a node of $X$ to a node of $Y$. For some root-node $r_{0} \in V$, an arborescence rooted at $r_{0}$ or an $r_{0}$-arborescence $(U, F)$ is a directed tree in which each node in $U$ is reachable from $r_{0}$. An arborescence is spanning if its node-set is $V$. We sometimes identify an arborescence $(U, F)$ with its arc-set $F$ and say that $F$ spans $U$. The nodeset of an $r$-arborescence $F$ is denoted by $V(F)$. It is possible that an $r_{0}$-arborescence has no arcs, in which case $V(F)=\left\{r_{0}\right\}$.

Let $\varrho(X)$ denote the in-degree of a set $X$. In [5], Edmonds gave the following characterization of the existence of $k$ pairwise arc-disjoint spanning $r_{0}$-arborescences.

TheOREm 1.1 (Edmonds). Let $D=(V, A)$ be a digraph with $r_{0} \in V$. There are $k$ pairwise arc-disjoint spanning $r_{0}$-arborescences in $D$ if and only if

$$
\varrho(X) \geq k \quad \text { for every } \emptyset \neq X \subseteq V-r_{0} .
$$

In the last couple of years, several extensions of Theorem 1.1 have been proved $[2,4,9,13]$. In [14], Katoh and Tanigawa considered the problem of packing rootedtrees in undirected graphs with additional matroid constraints. Durand de Gevigney, Nguyen, and Szigeti [4] later provided a directed counterpart of this result by characterizing digraphs having a packing of arborescences with matroid constraints. Recently, Király [15] gave the following generalization.

* Received by the editors November 20, 2015; accepted for publication (in revised form) June 30, 2016; published electronically September 1, 2016.

http://www.siam.org/journals/sidma/30-3/M104909.html

Funding: The research of the first and second authors was supported by the Hungarian Scientific Research Fund - OTKA, K109240. The second author's research was supported by the Bolyai Research Grant. The third author's research was supported by the JST, ERATO, Kawarabayashi Large Graph Project and by KAKENHI grants 24106002 and 24700004.

${ }^{\dagger}$ MTA-ELTE Egerváry Research Group, Department of Operations Research, Eötvös University, 1117 Budapest, Hungary (berkri@cs.elte.hu, tkiraly@cs.elte.hu).

$\ddagger$ University of Tsukuba, Tsukuba, Ibaraki, 305-8573, Japan (kobayashi@sk.tsukuba.ac.jp). 
Let $D=(V, A)$ be a digraph, and let $t$ be a positive integer that specifies the number of arborescences to pack. The set of integers from 1 to $t$ is denoted by $[t]$. We are also given a matroid $\mathcal{M}=([t], r)$ with rank function $r$ and a mapping $\pi:[t] \rightarrow V$ that specifies the roots of the arborescences. The triple $(D, \mathcal{M}, \pi)$ is called an $\mathcal{M}$ rooted digraph. For a set $X \subseteq V$, let $\pi^{-1}(X)=\{i \in[t]: \pi(i) \in X\}$. The mapping $\pi$ is $\mathcal{M}$-independent if $\pi^{-1}(v)$ is independent in $\mathcal{M}$ for every $v \in V$. The set of nodes from which a given set $X \subseteq V$ is reachable (including the nodes of $X$ themselves) is denoted by $\operatorname{Pred}_{X}$.

If an $\mathcal{M}$-rooted digraph $(D, \mathcal{M}, \pi)$ is given, we call a set of pairwise arc-disjoint arborescences $F_{1}, \ldots, F_{t}$ a maximal $\mathcal{M}$-independent packing of arborescences if $F_{i}$ is a $\pi(i)$-arborescence for every $i,\left\{i: v \in V\left(F_{i}\right)\right\}$ is independent in $\mathcal{M}$ for every $v \in V$, and $r\left(\left\{i: v \in V\left(F_{i}\right)\right\}\right)=r\left(\pi^{-1}\left(\operatorname{Pred}_{v}\right)\right)$ for every $v \in V$.

Theorem 1.2 (Király [15]). Let $(D, \mathcal{M}, \pi)$ be an $\mathcal{M}$-rooted digraph. There exists a maximal $\mathcal{M}$-independent packing of arborescences if and only if $\pi$ is $\mathcal{M}$-independent and

$$
\varrho(X) \geq r\left(\pi^{-1}\left(\operatorname{Pred}_{X}\right)\right)-r\left(\pi^{-1}(X)\right) \quad \text { for every } X \subseteq V .
$$

The proof in [15] is algorithmic, but it does not imply an algorithm for the following optimization problem: Given an $\mathcal{M}$-rooted digraph $(D, \mathcal{M}, \pi)$ and a cost function $c: A \rightarrow \mathbb{R}$, find a maximal $\mathcal{M}$-independent packing of arborescences that has minimum total cost. We will give a polynomial-time algorithm for this problem by solving a more general covering problem, related to another line of research on extensions of Edmonds's theorem. To describe these extensions, we need to introduce some notions concerning set families and bi-sets.

A family $\mathcal{F} \subseteq 2^{V}$ of subsets of a ground set $V$ is called intersecting if $X, Y \in$ $\mathcal{F}, X \cap Y \neq \emptyset$ implies $X \cap Y, X \cup Y \in \mathcal{F}$. Given a directed graph $D=(V, A)$, we say that an arc $a \in A$ covers a set $X \in \mathcal{F}$ if $a$ enters $X$, that is, the tail of $a$ is outside of $X$ while the head of $a$ is inside $X$. A subset of arcs $A^{\prime} \subseteq A$ covers an intersecting family $\mathcal{F}$ if each member of $\mathcal{F}$ is covered by at least one arc from $A^{\prime}$.

Frank [6] and Szegő [19] observed that Edmonds's theorem can be reformulated in terms of covering intersecting set families and thus gave abstract extensions of Edmonds's result. A bi-set counterpart of Szegö's theorem was proved in [2] by Frank and the first author of the present paper. A bi-set is a pair $X=\left(X_{I}, X_{O}\right)$ such that $X_{I} \subseteq X_{O} \subseteq V$, where $X_{I}$ and $X_{O}$ are called the inner set and the outer set of $X$, respectively. We will identify a bi-set $X=\left(X_{I}, X_{O}\right)$ for which $X_{O}=X_{I}$ with the set $X_{I}$, and hence the following notation can also be interpreted for sets. The set of all bi-sets on ground-set $V$ is denoted by $\mathcal{P}_{2}(V)=\mathcal{P}_{2}$. The intersection and union of bi-sets can be defined in a straightforward manner: for bi-sets $X$ and $Y$, we define $X \cap Y=\left(X_{I} \cap Y_{I}, X_{O} \cap Y_{O}\right)$ and $X \cup Y=\left(X_{I} \cup Y_{I}, X_{O} \cup Y_{O}\right)$. The notation $X \subseteq Y$ means that $X_{I} \subseteq Y_{I}$ and $X_{O} \subseteq Y_{O}$. Two bi-sets are intersecting if $X_{I} \cap Y_{I} \neq \emptyset$. A family $\mathcal{F}$ of bi-sets is called intersecting if $X, Y \in \mathcal{F}, X_{I} \cap Y_{I} \neq \emptyset$ implies $X \cap Y, X \cup Y \in \mathcal{F}$.

A bi-set function is a function $p: \mathcal{P}_{2} \rightarrow \mathbb{R}$. A bi-set function $p$ is fully supermodular (respectively, intersecting supermodular) if

$$
p(X)+p(Y) \leq p(X \cap Y)+p(X \cup Y)
$$

for every $X, Y \in \mathcal{P}_{2}$ (respectively, for every intersecting $X, Y \in \mathcal{P}_{2}$ ). If the reverse inequality holds, then $p$ is fully submodular. We call $p$ positively intersecting super- 
modular or positively intersecting submodular if the corresponding inequality holds whenever $X$ and $Y$ are intersecting and $p(X), p(Y)>0$.

Given a digraph $D=(V, A)$, an arc $a \in A$ enters or covers a bi-set $X$ if its head is in $X_{I}$ and its tail is outside $X_{O}$. A subset of arcs $A^{\prime} \subseteq A$ covers a bi-set family $\mathcal{F}$ if each member of $\mathcal{F}$ is covered by at least one arc from $A^{\prime}$. The set of arcs entering a bi-set $X$ is denoted by $\Delta^{i n}(X)$, while the number of $\operatorname{arcs}$ entering $X$ is denoted by $\varrho(X)$. The in-degree function $\varrho$ is a basic example for a submodular bi-set function. An arc is induced by bi-set $X$ if its tail is in $X_{O}$ and its head is in $X_{I}$. We say that the bi-set families $\mathcal{F}_{1}, \ldots, \mathcal{F}_{t}$ satisfy the mixed intersection property if $X \in \mathcal{F}_{i}, Y \in \mathcal{F}_{j}, X_{I} \cap Y_{I} \neq \emptyset$ implies $X \cap Y \in \mathcal{F}_{i} \cap \mathcal{F}_{j}$. The following theorem extends the result of Szegö to bi-set families.

Theorem 1.3 (Bérczi and Frank [2]). Let $D=(V, A)$ be a digraph, and let $\mathcal{F}_{1}, \ldots, \mathcal{F}_{t}$ be intersecting bi-set families satisfying the mixed intersection property. Then there exist pairwise disjoint arc-sets $A_{1}, \ldots, A_{t} \subseteq A$ such that $A_{i}$ covers $\mathcal{F}_{i}$ if and only if

$$
\varrho(X) \geq\left|I_{X}\right| \quad \text { for every } X \in \mathcal{P}_{2},
$$

where $I_{X}=\left\{i: X \in \mathcal{F}_{i}\right\}$.

Note that by using $I_{X}$, the mixed integer property can be rewritten as follows:

$$
I_{X} \subseteq I_{X \cap Y} \text { whenever } X, Y \in \mathcal{F} \text { and } X_{I} \cap Y_{I} \neq \emptyset \text {, }
$$

where $\mathcal{F}:=\bigcup_{i=1}^{t} \mathcal{F}_{i}$. Up to this point, we have two completely different generalizations of Edmonds's theorem. Theorem 1.2 is an extension of previous results on packing arborescences and characterizes the existence of maximal $\mathcal{M}$-independent arborescence packings. Meanwhile, Theorem 1.3 states the existence of disjoint covers of intersecting bi-set families and gives an abstract generalization of previous results. Our motivation was to find a common extension of these two results.

The rest of the paper is organized as follows. In section 2, we prove our main result: a characterization of arc-covers of intersecting bi-set families under matroid constraints. Section 3 presents some observations that follow from the proof. We also show how Theorems 1.2 and 1.3 can be derived from our results. In section 4, a polynomial-time algorithm is given for finding an arc-cover. In section 5 , we show that a minimum cost arc-cover can also be found in polynomial time using the ellipsoid method; moreover, if $\mathcal{F}$ is an intersecting family, then there is a strongly polynomial algorithm by reduction to submodular flows. Section 6 concludes the paper with a list of open problems related to packing arborescences.

\section{Characterization of valid arc-covers.}

2.1. Definitions and main theorem. As in Theorem 1.3, we consider a digraph $D=(V, A)$ and intersecting bi-set families $\mathcal{F}_{1}, \ldots, \mathcal{F}_{t}$ on the ground set $V$, and our aim is to find pairwise disjoint arc-sets $A_{1}, \ldots, A_{t} \subseteq A$ satisfying certain conditions. However, these conditions will be more subtle than the condition in Theorem 1.3 that $A_{i}$ must cover $\mathcal{F}_{i}$. Let $\mathcal{F}$ denote the family of bi-sets that appears in at least one $\mathcal{F}_{i}$, and for a bi-set $X \in \mathcal{F}$, let $I_{X}=\left\{i: X \in \mathcal{F}_{i}\right\}$. In addition, we are given a matroid $\mathcal{M}=([t], r)$, and a subset of indices $J_{X} \subseteq[t] \backslash I_{X}$ for every $X \in \mathcal{F}$. We want to find a family of pairwise disjoint arc-sets $A_{1}, \ldots, A_{t}$ of $A$ that satisfies

$$
r\left(J_{X} \cup\left\{i \in I_{X}: \varrho_{A_{i}}(X) \geq 1\right\}\right)=r\left(J_{X} \cup I_{X}\right) \quad \text { for every } X \in \mathcal{F},
$$


where $\varrho_{A_{i}}=\left|\Delta^{i n}(X) \cap A_{i}\right|$. A family of disjoint arc-sets $A_{1}, \ldots, A_{t} \subseteq A$ satisfying (2.1) is called a valid arc-cover. Intuitively, we may think of $[t]$ as a set of colors, the set $I_{X}$ as the colors required by bi-set $X$, and the set $J_{X}$ as the colors that $X$ already owns. The task is to color the arcs in such a way that for every bi-set $X$, the colors owned by $X$ and the required colors that enter $X$ together span the set of required colors in the matroid $\mathcal{M}$.

Finding a valid arc-cover in general is difficult (see the end of this subsection for hardness results), so we need to add additional conditions on the set families, analogously to the mixed intersection property in Theorem 1.3. The closure of a set $J \subseteq[t]$ in $\mathcal{M}$ is denoted by $\operatorname{Span}(J)$, that is, $\operatorname{Span}(J)=\{i: r(J+i)=r(J)\}$. A set $J \subseteq[t]$ is closed if $\operatorname{Span}(J)=J$. We introduce the following bi-set function defined on $\mathcal{P}_{2}$ :

$$
p(X)= \begin{cases}r\left(I_{X} \cup J_{X}\right)-r\left(J_{X}\right) & \text { if } X \in \mathcal{F}, \\ 0 & \text { otherwise }\end{cases}
$$

A bi-set $X$ is said to be active if $p(X)>0$, which is equivalent to $I_{X} \backslash \operatorname{Span}\left(J_{X}\right) \neq \emptyset$. We denote the set $I_{X} \backslash \operatorname{Span}\left(J_{X}\right)$ by $I_{X}^{a c t}$ and say that $X$ is active for $i$ if $i \in I_{X}^{\text {act }}$. Note that all active bi-sets are in $\mathcal{F}$. We say that the active intersection property is satisfied if

$$
I_{X}^{a c t} \subseteq I_{X \cap Y}^{a c t} \text { whenever } X, Y \in \mathcal{F} \text { and } X_{I} \cap Y_{I} \neq \emptyset .
$$

Note that if $\mathcal{M}$ is the free matroid on $[t]$, then $I_{X}^{a c t}=I_{X}$, which means that (AIP) coincides with (MIP). In this sense, we can say that (AIP) is a matroidal extension of (MIP).

Our first result is the following characterization of valid arc-covers for the case when $I_{X} \cup J_{X}=[t]$ for every $X \in \mathcal{F}$.

Theorem 2.1. Let $\mathcal{M}=([t], r)$ be a matroid and $D=(V, A)$ be a digraph. Let $\mathcal{F}_{1}, \ldots, \mathcal{F}_{t}$ be intersecting bi-set families on ground set $V$, let $\mathcal{F}=\bigcup_{i=1}^{t} \mathcal{F}_{i}$, and let $J_{X}:=[t] \backslash I_{X}$ for every $X \in \mathcal{F}$, satisfying (AIP). Then a valid arc-cover exists if and only if

$$
\varrho(X) \geq r([t])-r\left(J_{X}\right) \quad \text { for every } X \in \mathcal{F} .
$$

As we will see in section 3 , this theorem generalizes Theorem 1.3. However, we cannot derive Theorem 1.2 from this theorem, which motivates us to consider a further generalization. Indeed, we generalize the condition $I_{X} \cup J_{X}=[t]$ to the following index union property.

$$
I_{X^{\prime}} \cup J_{X^{\prime}}=I_{X} \cup J_{X} \text { whenever } X, X^{\prime} \in \mathcal{F} \text { and } X^{\prime} \subseteq X .
$$

Our main result is stated as follows.

Theorem 2.2. Let $\mathcal{M}=([t], r)$ be a matroid and $D=(V, A)$ be a digraph. Let $\mathcal{F}_{1}, \ldots, \mathcal{F}_{t}$ be intersecting bi-set families on ground set $V$, let $\mathcal{F}=\bigcup_{i=1}^{t} \mathcal{F}_{i}$, and let $J_{X} \subseteq[t] \backslash I_{X}$ for every $X \in \mathcal{F}$, satisfying (AIP) and (IUP). Then a valid arc-cover exists if and only if

$$
\varrho(X) \geq r\left(I_{X} \cup J_{X}\right)-r\left(J_{X}\right) \quad \text { for every } X \in \mathcal{F} .
$$


Since Theorem 2.1 is a special case of this theorem, it suffices to prove Theorem 2.2. The proof is presented in sections 2.2 and 2.3 .

To explain the need for property (IUP), we show that NP-hard problems are obtained if equality is replaced by " $\subseteq$ " or " $\supseteq$ " in (IUP). First, let us consider the case when (IUP) is replaced by the condition that $I_{X^{\prime}} \cup J_{X^{\prime}} \subseteq I_{X} \cup J_{X}$ whenever $X, X^{\prime} \in \mathcal{F}$ and $X^{\prime} \subseteq X$. Let $D=(V, A)$ be a directed graph, let $t=4$, and $\mathcal{M}=([4], r)$ let be the matroid with cycles $\{1,3\}$ and $\{2,4\}$. Define $\mathcal{F}_{1}=\mathcal{F}_{2}=2^{V} \backslash\{\emptyset\}$ and $\mathcal{F}_{3}=\mathcal{F}_{4}=\emptyset$. Finally, let $J_{V}=\{3,4\}$, and let $J_{X}$ be empty otherwise. It is easy to check that (AIP) and the relaxed version of (IUP) are satisfied. By definition, an arc-cover is valid if and only if $A_{1}$ and $A_{2}$ are both strongly connected spanning subdigraphs; but it is NP-complete to decide whether a digraph can be decomposed into two strongly connected spanning subdigraphs [1, Theorem 13.10.1].

What if (IUP) is replaced by $I_{X^{\prime}} \cup J_{X^{\prime}} \supseteq I_{X} \cup J_{X}$ ? Let $D=(V, A)$ be a directed graph with a root node $r_{0}$ and a terminal set $T \subseteq V-r_{0}$. The problem of deciding whether $D$ can be decomposed into two subdigraphs so that every terminal is reachable from $r_{0}$ in both of them is NP-complete, since the two disjoint dipaths problem can be reduced to it. To formulate this problem in our setting, set $t=3$ and let $\mathcal{M}=([3], r)$ be the matroid with cycle $\{1,2,3\}$. Define $\mathcal{F}_{1}=\mathcal{F}_{2}=2^{V-r_{0}} \backslash\{\emptyset\}$ and $\mathcal{F}_{3}=\emptyset$. Let $J_{X}=\{3\}$ if $X \cap T=\emptyset$, and $J_{X}=\emptyset$ otherwise. With these definitions (AIP) holds because $I_{X}^{a c t}=\{1,2\}$ for every $X \in \mathcal{F}$, and also the relaxed version of (IUP) holds. An arc-cover is valid if and only if $A_{1} \cup A_{2}$ contains a spanning arborescence rooted at $r_{0}$, and every $v \in T$ is reachable from $r_{0}$ in both $A_{1}$ and $A_{2}$. This is equivalent to the original problem, since we can assume without loss of generality that $D$ contains a spanning arborescence rooted at $r_{0}$.

2.2. Preliminary observations. Assume that (AIP) and (IUP) hold. We start with an easy observation on the properties of $J_{X}$.

Proposition 2.3. If $X, X^{\prime} \in \mathcal{F}$ and $X^{\prime} \subseteq X$, then $J_{X^{\prime}} \subseteq \operatorname{Span}\left(J_{X}\right)$.

Proof. Property (IUP) implies that $I_{X^{\prime}} \cup J_{X^{\prime}}=I_{X} \cup J_{X}$. If $X$ is not active, then $\operatorname{Span}\left(J_{X}\right) \supseteq I_{X} \cup J_{X} \supseteq J_{X^{\prime}}$, so we are done. If $X$ is active, then by (AIP) for $X$ and $X^{\prime}$ we have $I_{X^{\prime}} \backslash \operatorname{Span}\left(J_{X^{\prime}}\right) \supseteq I_{X} \backslash \operatorname{Span}\left(J_{X}\right)$, which, together with $I_{X^{\prime}} \cup J_{X^{\prime}}=I_{X} \cup J_{X}$, implies that $J_{X^{\prime}} \subseteq \operatorname{Span}\left(J_{X}\right)$.

From this observation we can derive the positively intersecting supermodularity of the bi-set function $p$ defined in (2.2).

Proposition 2.4. The bi-set function $p$ is positively intersecting supermodular.

Proof. Let $X, Y$ be intersecting bi-sets with $p(X)>0, p(Y)>0$. Both $X$ and $Y$ are active, and so $X \cap Y \in \mathcal{F}$ by (AIP). Note that Proposition 2.3 implies $J_{X \cap Y} \subseteq \operatorname{Span}\left(J_{X}\right) \cap \operatorname{Span}\left(J_{Y}\right)$.

Assume first that $X \cup Y \in \mathcal{F}$. As the $\mathcal{F}_{i}$ 's are intersecting, $I_{X \cup Y} \supseteq I_{X} \cap I_{Y}$ holds. This, together with (IUP) and the disjointness of $I_{Z}$ and $J_{Z}$ for every $Z \in \mathcal{F}$, implies $J_{X \cup Y} \subseteq J_{X} \cup J_{Y}$. Thus we have, by the submodularity of $r$,

$$
\begin{aligned}
r\left(J_{X}\right)+r\left(J_{Y}\right) & =r\left(\operatorname{Span}\left(J_{X}\right)\right)+r\left(\operatorname{Span}\left(J_{Y}\right)\right) \\
& \geq r\left(\operatorname{Span}\left(J_{X}\right) \cap \operatorname{Span}\left(J_{Y}\right)\right)+r\left(\operatorname{Span}\left(J_{X}\right) \cup \operatorname{Span}\left(J_{Y}\right)\right) \\
& \geq r\left(J_{X \cap Y}\right)+r\left(J_{X \cup Y}\right) .
\end{aligned}
$$


Using this and (IUP), we obtain

$$
\begin{aligned}
p(X)+p(Y) & =r\left(I_{X} \cup J_{X}\right)-r\left(J_{X}\right)+r\left(I_{Y} \cup J_{Y}\right)-r\left(J_{Y}\right) \\
& \leq r\left(I_{X \cap Y} \cup J_{X \cap Y}\right)-r\left(J_{X \cap Y}\right)+r\left(I_{X \cup Y} \cup J_{X \cup Y}\right)-r\left(J_{X \cup Y}\right) \\
& =p(X \cap Y)+p(X \cup Y) .
\end{aligned}
$$

Now assume that $X \cup Y \notin \mathcal{F}$. By (IUP), $I_{X} \cup J_{X}=I_{X \cap Y} \cup J_{X \cap Y}=I_{Y} \cup J_{Y}$; let $q$ denote the rank of this set. As the $\mathcal{F}_{i}$ 's are intersecting, we have $I_{X} \cap I_{Y}=\emptyset$, which implies $I_{X} \subseteq J_{Y}$. Hence we have, by the submodularity of $r$ and $X \cup Y \notin \mathcal{F}$,

$$
\begin{aligned}
p(X)+p(Y) & =2 q-r\left(J_{X}\right)-r\left(J_{Y}\right) \\
& =2 q-r\left(\operatorname{Span}\left(J_{X}\right)\right)-r\left(\operatorname{Span}\left(J_{Y}\right)\right) \\
& \leq 2 q-r\left(\operatorname{Span}\left(J_{X}\right) \cap \operatorname{Span}\left(J_{Y}\right)\right)-r\left(\operatorname{Span}\left(J_{X}\right) \cup \operatorname{Span}\left(J_{Y}\right)\right) \\
& \leq q-r\left(\operatorname{Span}\left(J_{X}\right) \cap \operatorname{Span}\left(J_{Y}\right)\right) \\
& \leq q-r\left(J_{X \cap Y}\right) \\
& =p(X \cap Y)+p(X \cup Y) .
\end{aligned}
$$

2.3. Proof of Theorem 2.2. Observe that (2.4) is equivalent to $\varrho(X) \geq p(X)$ for every $X \in \mathcal{F}$. A bi-set $X$ is called tight if $\varrho(X)=p(X)>0$. A bi-set is tight for $i$ if it is tight and $i \notin I_{X}^{a c t}$. By definition, tight bi-sets are in $\mathcal{F}$. The core of the proof is the following reduction step.

Definition 2.5 (reduction on $(X, a, i)$ ). Suppose that $X \in \mathcal{F}, i \in I_{X}^{a c t}$, and $a \in \Delta^{i n}(X)$ are such that $a$ is not induced by any bi-set active for $i$ and does not enter any bi-set tight for $i$. Then the triple $(X, a, i)$ is called reducible. A reduction on $(X, a, i)$ consists of

- adding a to $A_{i}$ and removing it from $A$;

- adding $i$ to $J_{Z}$ for every bi-set $Z$ covered by a; and

- removing bi-sets covered by a from $\mathcal{F}_{i}$.

We usually use the notation $D^{\prime}, \mathcal{F}_{i}^{\prime}, I_{Z}^{\prime}$, and $J_{Z}^{\prime}$ for the state after the reduction.

Lemma 2.6. A reduction step on $(X, a, i)$ preserves the conditions of the theorem.

Proof. Let $\mathcal{F}^{\prime}$ denote the family of bi-sets in $\mathcal{F}$ that have not been removed, and let $\varrho^{\prime}$ be the in-degree function of the digraph obtained by deleting arc $a$. The family $\mathcal{F}_{i}^{\prime}$ is intersecting because if $U \in \mathcal{F}_{i}$ and $W \in \mathcal{F}_{i}$ are not covered by $a$, then neither are their intersection $U \cap W$ and their union $U \cup W$.

The validity of (AIP) can be seen as follows. Assume that $U \in \mathcal{F}_{i}^{\prime}$ with $i \in I_{U}^{a c t}$ and $W \in \mathcal{F}$. As only $\mathcal{F}_{i}$ changes during this step, it suffices to show that if $U$ and $W$ are intersecting and $U$ remains active for $i$ after the reduction, then so does $U \cap W$. If this does not hold, then $a$ covers $U \cap W$ but does not cover $U$, and hence it is induced by $U$. However, we assumed that $a$ is not induced by a bi-set active for $i$, a contradiction.

Note that $I_{Z} \cup J_{Z}$ does not change during the reduction for any bi-set $Z \in \mathcal{F}$, and hence (IUP) remains valid. Finally, $\varrho^{\prime}(Z) \geq r\left(I_{Z}^{\prime} \cup J_{Z}^{\prime}\right)-r\left(J_{Z}^{\prime}\right)$ holds for each $Z \in \mathcal{F}^{\prime}$, as we assumed that $a$ enters no bi-set that is tight for $i$.

We prove the theorem by induction on $\sum_{Z \in \mathcal{F}} p(Z)$. If $\mathcal{F}=\emptyset$ or $p(Z)=0$ for each $Z \in \mathcal{F}$, then (2.1) is clearly satisfied and we are done. Otherwise, take an inclusionwise maximal bi-set $X$ in $\mathcal{F}$ with $p(X) \geq 1$. From now on, our aim is to show that there exists a triple $(X, a, i)$ satisfying the conditions of Definition 2.5. This would prove the theorem by induction. 
The following can be derived using the maximality of $X$.

Proposition 2.7. There is no bi-set $Z$ intersecting $X$ such that $Z$ is active for every $i \in I_{X}^{a c t}$ and $X \cup Z$ is strictly larger than $X$.

Proof. Suppose for contradiction that such a $Z$ exists. As $I_{X}^{a c t} \cap I_{Z}^{a c t} \neq \emptyset$ and the $\mathcal{F}_{i}$ 's are intersecting, $X \cup Z \in \mathcal{F}$. Moreover, $I_{X}^{a c t} \subseteq I_{Z}^{a c t}$, so, by $\mathcal{F}_{i}$ 's being intersecting, $I_{X} \backslash \operatorname{Span}\left(J_{X}\right) \subseteq I_{X \cup Z}$. By (IUP), $I_{X} \cup J_{X}=I_{X \cup Z} \cup J_{X \cup Z}$, so $J_{X \cup Z}=$ $\left(I_{X} \cup J_{X}\right) \backslash I_{X \cup Z} \subseteq \operatorname{Span}\left(J_{X}\right)$. Hence we have

$$
\begin{aligned}
p(X \cup Z) & =r\left(I_{X \cup Z} \cup J_{X \cup Z}\right)-r\left(J_{X \cup Z}\right) \\
& \geq r\left(I_{X} \cup J_{X}\right)-r\left(J_{X}\right) \\
& \geq 1,
\end{aligned}
$$

contradicting the maximality of $X$.

In what follows, we distinguish two cases.

Case 1. There is a tight bi-set $Y \in \mathcal{F}$ intersecting $X$ with $I_{X}^{\text {act }} \backslash I_{Y}^{a c t} \neq \emptyset$.

Let $Y \in \mathcal{F}$ be inclusionwise minimal among these tight bi-sets.

Proposition 2.8. There is an arc in $\Delta^{i n}(X \cap Y) \backslash \Delta^{i n}(Y)$.

Proof. Take any index $i \in I_{X}^{a c t} \backslash I_{Y}^{a c t}$. As $X$ is active for $i$, (AIP) implies that $X \cap Y$ is also active for $i$, and thus $i \in I_{X \cap Y}^{a c t}=I_{X \cap Y} \backslash \operatorname{Span}\left(J_{X \cap Y}\right)$. Property (IUP) shows that $i \in I_{Y} \cup J_{Y}$. But $i \notin I_{Y}^{a c t}$, so $i \in \operatorname{Span}\left(J_{Y}\right) \backslash \operatorname{Span}\left(J_{X \cap Y}\right)$. This, together with Proposition 2.3, gives $r\left(J_{Y}\right)>r\left(J_{X \cap Y}\right)$.

By tightness of $Y$, the definition of $p$, (IUP), the above, and (2.4), we have

$$
\begin{aligned}
\varrho(Y) & =p(Y) \\
& =r\left(J_{Y} \cup I_{Y}\right)-r\left(J_{Y}\right) \\
& <r\left(J_{X \cap Y} \cup I_{X \cap Y}\right)-r\left(J_{X \cap Y}\right) \\
& \leq \varrho(X \cap Y) ;
\end{aligned}
$$

therefore there is an arc $a \in \Delta^{i n}(X \cap Y) \backslash \Delta^{i n}(Y)$.

Choose an arc $a$ provided by Proposition 2.8.

Proposition 2.9. There is no bi-set $W$ such that $a \in \Delta^{i n}(W)$ and $W$ is tight for some index in $I_{X}^{a c t} \backslash I_{Y}^{a c t}$.

Proof. Suppose for contradiction that there is a bi-set $W$ that is tight for some $i \in I_{X}^{a c t} \backslash I_{Y}^{a c t}$. Since $a$ enters $W$, it is a tight bi-set that intersects $Y$. By tightness of $Y$ and $W$, Proposition 2.4, and (2.4), we have

$$
\begin{aligned}
\varrho(Y)+\varrho(W) & =p(Y)+p(W) \\
& \leq p(Y \cap W)+p(Y \cup W) \\
& \leq \varrho(Y \cap W)+\varrho(Y \cup W) .
\end{aligned}
$$

As the in-degree function $\varrho$ is submodular, we have equality throughout, which has two important consequences. First, $p(X \cap Y)=\varrho(X \cap Y)$ and also $\varrho(X \cap Y) \geq 1$ due to $\operatorname{arc} a$. Second, the proof of Proposition 2.4 shows that $r\left(J_{Y \cap W}\right)=r\left(\operatorname{Span}\left(J_{Y}\right) \cap\right.$ $\left.\operatorname{Span}\left(J_{W}\right)\right)$ must hold, which, together with Proposition 2.3, implies $\operatorname{Span}\left(J_{Y \cap W}\right)=$ $\operatorname{Span}\left(J_{Y}\right) \cap \operatorname{Span}\left(J_{W}\right)$. We know by (IUP) that $I_{Y} \cup J_{Y}=I_{Y \cap W} \cup J_{Y \cap W}=I_{W} \cup J_{W}$. These together give $I_{Y \cap W} \backslash \operatorname{Span}\left(J_{Y \cap W}\right)=\left(I_{Y} \backslash \operatorname{Span}\left(J_{Y}\right)\right) \cup\left(I_{W} \backslash \operatorname{Span}\left(J_{W}\right)\right)$, i.e., $I_{Y \cap W}^{a c t}=I_{Y}^{a c t} \cup I_{W}^{a c t}$. We assumed that there is an index $i$ in $I_{X}^{a c t} \backslash\left(I_{Y}^{a c t} \cup I_{W}^{a c t}\right)$, so there is an index $i \in I_{X}^{a c t}$ for which $Y \cap W$ is tight, contradicting the choice of $Y$. 
Proposition 2.10. There is an index $i \in I_{X}^{a c t}$ such that $(X, a, i)$ is reducible.

Proof. We know by Proposition 2.9 that $a$ does not enter any bi-set tight for any $i \in I_{X}^{a c t} \backslash I_{Y}^{a c t}$. Suppose that for each $i \in I_{X}^{a c t} \backslash I_{Y}^{a c t}$ there is a bi-set $Z_{i}$ active for $i$ that induces $a$. Then, by (AIP), $Z^{\prime}=\bigcap Z_{i}$ is a bi-set that is active for every $i \in I_{X}^{a c t} \backslash I_{Y}^{a c t}$. On the other hand, $Y$ is active for every $i \in I_{Y}^{a c t}$ and the tail of $a$ is in $Z=Y \cap Z^{\prime}$, so, by (AIP), $Z$ is active for every $i \in I_{X}^{a c t}, Z$ intersects $X$, and $X \cup Z$ is strictly larger than $X$, contradicting Proposition 2.7.

We have proved the existence of a reducible triple $(X, a, i)$, so Case 1 is settled.

Case 2. There is no tight bi-set $Y \in \mathcal{F}$ with $I_{X}^{\text {act }} \backslash I_{Y}^{a c t} \neq \emptyset$ intersecting $X$.

Take an arbitrary $\operatorname{arc} a$ entering $X$. Such an arc exists by $(2.4)$ and $p(X) \geq 1$. Suppose that for each $i \in I_{X}^{a c t}$ there is a bi-set $Z_{i}$ which is active for $i$ and induces $a$. By (AIP), $Z=\bigcap Z_{i}$ is active for each $i \in I_{X}^{a c t}, Z$ intersects $X$, and $X \cup Z$ is strictly larger than $X$, contradicting Proposition 2.7. Thus there is an index $i \in I_{X}^{\text {act }}$ such that $(X, a, i)$ is reducible, concluding the proof.

3. Consequences and special cases. From the proof in the previous section we can derive the following strengthening of Theorem 2.2.

Corollary 3.1. The valid arc-cover $A_{1}, \ldots, A_{t}$ provided by the proof of Theorem 2.2 satisfies the following for each minimal $M \in \mathcal{F}$ :

1. $\varrho_{A_{i}}(M) \leq 1$ for $1 \leq i \leq t$.

2. If $J_{M}$ is independent, then $J_{M} \cup\left\{i: \varrho_{A_{i}}(M) \geq 1\right\}$ is also independent.

Proof. Consider the addition of an $\operatorname{arc} a$ to an $\operatorname{arc}$ set $A_{j}$. According to the proof, $a$ enters a bi-set $X \in \mathcal{F}_{j}$ which is active for $j$ and inclusionwise maximal. If $M$ is a minimal set in $\mathcal{F}$ entered by $a$, then, by (AIP), $M \cap X \in \mathcal{F}_{j}$, so, by the minimality of $M, M \subseteq X$. If $M$ was already covered by an arc $a^{\prime} \in A_{j}$ at this point, then $a^{\prime}$ is induced by $X$ as $X$ is active for $j$, in contradiction to the way we chose $a^{\prime}$. Thus item 1 indeed holds. Furthermore, the rank of $J_{M} \cup\left\{i: \varrho_{A_{i}}(M) \geq 1\right\}$ increases by 1 due to the addition of index $j$, since $M$ is active for $j$. Therefore at the end of the algorithm $J_{M} \cup\left\{i: \varrho_{A_{i}}(M) \geq 1\right\}$ is independent unless $J_{M}$ itself was dependent.

As was mentioned earlier, previous results on packing arborescences can be derived from Theorem 2.2. First we show that it is an extension of Theorem 1.3.

Proof of Theorem 1.3. The "only if" part can be seen easily, and hence it suffices to prove the "if" part. For $X \in \mathcal{P}_{2}$, let $J_{X}=[t] \backslash I_{X}$; property (IUP) is obviously satisfied. That is, we consider the case as in Theorem 2.1.

Let $\mathcal{M}=([t], r)$ be the free matroid on $[t]$. For a bi-set $X \in \mathcal{P}_{2}$ we have $r\left(I_{X} \cup\right.$ $\left.J_{X}\right)-r\left(J_{X}\right)=\left|\left\{i: X \in \mathcal{F}_{i}\right\}\right|$, and thus condition (2.4) of Theorem 2.2 holds. Note that $I_{X}^{a c t}=I_{X}$ in this case and $r(S)=|S|$ for each $S \subseteq[t]$. Moreover, (MIP) implies that property (AIP) is also satisfied.

By the above, Theorem 2.2 ensures the existence of pairwise disjoint arc-sets such that

$$
\begin{aligned}
\left|J_{X}\right|+\left|\left\{i: X \in \mathcal{F}_{i}, \varrho_{A_{i}}(X) \geq 1\right\}\right| & =r\left(J_{X} \cup\left\{i \in I_{X}^{a c t}: \varrho_{A_{i}}(X) \geq 1\right\}\right) \\
& =r\left(I_{X} \cup J_{X}\right) \\
& =\left|J_{X}\right|+\left|\left\{i: X \in \mathcal{F}_{i}\right\}\right| .
\end{aligned}
$$

That is, $\mathcal{F}_{i}$ is covered by $A_{i}$, as required.

It would be natural to expect that Theorem 2.2 generalizes Theorem 1.2 similarly to the way Theorem 1.3 did for the results of Kamiyama, Katoh, and Takizawa [13] 
and Fujishige [9]. However, the presence of matroidal constraints calls for caution. The problem is that a valid arc-cover does not necessarily consist of arborescences. For example, if the matroid has two parallel elements $i$ and $j$, then we can move arcs between $A_{i}$ and $A_{j}$ without affecting the validity of the arc-cover, so the algorithm described in the previous section does not distinguish between $A_{i}$ and $A_{j}$. Therefore we need to refine the choice of the reduction step in the proof of Theorem 2.2 in order to obtain arborescences.

Proof of Theorem 1.2. The "only if" part can be seen easily, and hence it suffices to show the "if" part. Let $(D=(V, A), \mathcal{M}=([t], r), \pi)$ be an $\mathcal{M}$-rooted digraph; we assume that $\pi$ is $\mathcal{M}$-independent and (1.2) holds. Let $r_{i}=\pi(i)$, and let $U_{i}$ be the set of nodes reachable from $r_{i}$ in $D$. The sets $U_{i}$ define a partition of $V$ into atoms: two nodes $u$ and $v$ belong to the same atom if there is no $U_{i}$ with $\left|\{u, v\} \cap U_{i}\right|=1$. A subset of an atom is called a subatom. Let

$$
\begin{aligned}
\overline{\mathcal{P}}_{2}=\left\{X \in \mathcal{P}_{2}: X_{I}\right. \text { is a nonempty subatom, } \\
\left.X_{O} \backslash X_{I} \text { does not intersect the atom containing } X_{I}\right\} .
\end{aligned}
$$

Define the bi-set families $\mathcal{F}_{1}, \ldots, \mathcal{F}_{t}$ as follows:

$$
\mathcal{F}_{i}=\left\{X \in \overline{\mathcal{P}}_{2}: X_{I} \subseteq U_{i}-r_{i}, X_{O} \cap U_{i}=X_{I}\right\} .
$$

For $X \in \overline{\mathcal{P}}_{2}$, let $J_{X}=\left\{i: X_{I} \subseteq U_{i}, X \notin \mathcal{F}_{i}\right\}$. Note that bi-sets of the form $(W, W)$, where $W \subseteq U_{i}-r_{i}$ is a subatom, are contained in $\mathcal{F}_{i}$. In this case we use $I_{W}$ and $J_{W}$ for denoting the corresponding sets of indices. Clearly, $I_{W}=\left\{i: W \subseteq U_{i}, r_{i} \notin W\right\}$ and $J_{W}=\left\{i: W \subseteq U_{i}, r_{i} \in W\right\}$.

It is easy to check that the $\mathcal{F}_{i}$ 's are intersecting bi-set families. For a bi-set $X \in \mathcal{F}=\bigcup_{i=1}^{t} \mathcal{F}_{i}, I_{X} \cup J_{X}=\left\{i: X_{I} \subseteq U_{i}\right\}$, so (IUP) in Theorem 2.2 is clearly satisfied. Assume that $X, X^{\prime} \in \mathcal{F}$ and $X^{\prime} \subseteq X$. By the definition of $J_{X}$, we have $J_{X^{\prime}} \subseteq J_{X}$. This, together with (IUP), implies $I_{X}^{a c t} \subseteq I_{X^{\prime}}^{a c t}$, and hence (AIP) also holds.

We claim that condition (2.4) in Theorem 2.2 is also satisfied. Indeed, for a given bi-set $X \in \mathcal{F}$ let $Y$ be the bi-set with

$$
\begin{aligned}
Y_{I} & =X_{I}, \\
Y_{O} \backslash Y_{I} & =V \backslash\left(\bigcup_{i \in I_{X} \cup J_{X}} U_{i}\right) .
\end{aligned}
$$

Then $I_{Y} \cup J_{Y}=I_{X} \cup J_{X}, J_{Y} \subseteq J_{X}$, and $\Delta^{i n}(Y) \subseteq \Delta^{i n}(X)$ (although $X_{O} \subseteq Y_{O}$ does not necessarily hold). Note that each arc entering $Y_{O}$ enters $Y_{I}$, and hence we have

$$
\begin{aligned}
r\left(I_{X} \cup J_{X}\right)-r\left(J_{X}\right) & \leq r\left(I_{Y} \cup J_{Y}\right)-r\left(J_{Y}\right) \\
& =r\left(\pi^{-1}\left(\operatorname{Pred}_{Y_{O}}\right)\right)-r\left(\pi^{-1}\left(Y_{O}\right)\right) \\
& \leq \varrho\left(Y_{O}\right)=\varrho(Y) \leq \varrho(X),
\end{aligned}
$$

proving (2.4).

The above means that the conditions of Theorem 2.2 hold, so there are pairwise disjoint arc-sets $A_{1}, \ldots, A_{t}$ such that

$$
r\left(J_{X} \cup\left\{i: \varrho_{A_{i}}(X) \geq 1\right\}\right)=r\left(I_{X} \cup J_{X}\right)
$$


for every $X \in \mathcal{F}$. We claim that these arc-sets can be chosen such that $A_{i}$ is an arborescence rooted at $r_{i}$. In order to prove this, we have to choose the reducible triples $(X, a, i)$ more carefully than in the proof of Theorem 2.2 .

Consider a general step of the proof, and assume that the sets of arcs that were already added to $A_{1}, \ldots, A_{t}$ form arborescences. At this point, the sets $I_{X}, J_{X}$, $\mathcal{F}_{i}$, and $\mathcal{F}$ have already been modified. We distinguish the modified sets from their original versions by adding a bar above the usual notation. Thus

$$
\begin{aligned}
& \bar{I}_{X}=\left\{i: X_{I} \subseteq U_{i} \backslash V\left(A_{i}\right), X_{O} \cap U_{i}=X_{I}\right\}, \\
& \bar{J}_{X}=\left\{i: X_{I} \subseteq U_{i}, i \notin \bar{I}_{X}\right\} .
\end{aligned}
$$

In particular, for a subatom $W \subseteq V$ we have $\bar{I}_{W}=\left\{i: W \subseteq U_{i}, W \cap V\left(A_{i}\right)=\emptyset\right\}$ and $\bar{J}_{W}=\left\{i: W \cap V\left(A_{i}\right) \neq \emptyset\right\}$.

Proposition 3.2. If $V\left(A_{i}\right) \cap X_{O} \neq \emptyset$ for a bi-set $X \in \overline{\mathcal{F}}$, then $X$ is not active for $i$.

Proof. Since $X_{I}$ is a subatom, either $X_{I} \cap U_{i}=\emptyset$ or $X_{I} \subseteq U_{i}$ holds. We consider the following three cases:

- If $X_{I} \cap U_{i}=\emptyset$, then $i \notin \bar{I}_{X} \cup \bar{J}_{X}$.

- If $X_{I} \subseteq U_{i}$ and $V\left(A_{i}\right) \cap X_{I} \neq \emptyset$, then $i \in \bar{J}_{X}$.

- If $X_{I} \subseteq U_{i}$ and $V\left(A_{i}\right) \cap X_{I}=\emptyset$, then, by $V\left(A_{i}\right) \cap X_{O} \neq \emptyset, X_{O} \cap U_{i} \neq X_{I}$, which implies that $i \in \bar{J}_{X}$.

In each case, by definition, the proposition follows.

To select a reducible triple $(X, a, i)$, first we choose an active bi-set $X$ that is inclusionwise maximal among those for which $\left|\bar{I}_{X} \cup \bar{J}_{X}\right|$ is minimal.

Proposition 3.3. For every arc $u v \in \Delta(X)$, node $u$ is reached in some $A_{j}$ for which $j \in \bar{I}_{X}^{a c t}$.

Proof. We distinguish two cases based on whether $u$ and $v$ are in the same atom.

Case 1 . Nodes $u$ and $v$ are in the same atom.

Consider the bi-set $X^{\prime}=\left(X_{I}+u, X_{O}+u\right)$. Note that $\bar{I}_{X} \cup \bar{J}_{X}=\bar{I}_{X^{\prime}} \cup \bar{J}_{X^{\prime}}=\bar{I}_{u} \cup \bar{J}_{u}$ and we have $\bar{I}_{X^{\prime}}=\bar{I}_{X} \backslash \bar{J}_{u}$ and $\bar{J}_{X^{\prime}}=\bar{J}_{X} \cup \bar{J}_{u}$. If there is an index $j \in \bar{I}_{X}^{a c t} \cap \bar{J}_{u}$, then $u$ is reached in $A_{j}$, and we are done. Otherwise, $\bar{J}_{u} \backslash \operatorname{Span}\left(\bar{J}_{X}\right)=\emptyset$, implying $\bar{I}_{X^{\prime}}^{a c t}=\bar{I}_{X}^{a c t} \neq \emptyset$, which contradicts the maximality of $X$.

Case 2. Nodes $u$ and $v$ lie in different atoms.

In this case $\bar{I}_{u} \cup \bar{J}_{u} \subset \bar{I}_{X} \cup \bar{J}_{X}$ because of the arc $u v$. By the choice of $X$, the biset $(u, u)$ is not active, so $\bar{I}_{u} \subseteq \operatorname{Span}\left(\bar{J}_{u}\right)$. The bi-set $X^{\prime \prime}=\left(X_{I}, X_{O}+u\right)$ is also inactive because of the maximality of $X$. We have $\bar{J}_{X^{\prime \prime}}=\bar{J}_{X} \cup \bar{I}_{u} \cup \bar{J}_{u}$, and thus $\operatorname{Span}\left(\bar{J}_{X^{\prime \prime}}\right)=\operatorname{Span}\left(\bar{J}_{X} \cup \bar{J}_{u}\right)$. If there is an index $j \in \bar{I}_{X}^{\text {act }} \cap \bar{J}_{u}$, then $u$ is reached in $A_{j}$ and we are done. Otherwise, we have $\bar{J}_{u} \subseteq \operatorname{Span}\left(\bar{J}_{X}\right)$, and hence $\operatorname{Span}\left(\bar{J}_{X^{\prime \prime}}\right)=\operatorname{Span}\left(\bar{J}_{X} \cup \bar{J}_{u}\right)=\operatorname{Span}\left(\bar{J}_{X}\right)$, which contradicts that $X$ is active and $X^{\prime \prime}$ is inactive.

As in the proof of Theorem 2.2, we consider two cases. Assume first that there is a tight bi-set $Y \in \overline{\mathcal{F}}$ intersecting $X$ with $\bar{I}_{X}^{a c t} \backslash \bar{I}_{Y}^{a c t} \neq \emptyset$, and choose a minimal tight bi-set $Y$ with this property. By Proposition 2.8, there is an arc $u v=a \in \Delta^{i n}(X)$ induced by $Y$. By Proposition 3.3, $u$ is reached in some $A_{j}$ such that $j \in \bar{I}_{X}^{a c t}$. By Proposition 3.2, we have $v \notin V\left(A_{j}\right), j \in \bar{I}_{X}^{a c t} \backslash \bar{I}_{Y}^{a c t}$ and that $a$ is not induced by a bi-set $Z$ active for $j$.

If there is no such tight bi-set, then we can choose an $\operatorname{arc} u v=a \in \Delta^{i n}(X)$ arbitrarily. By Proposition $3.3, u$ is reached in some $A_{j}$ such that $j \in \bar{I}_{X}^{a c t}$ and by 
Proposition 3.2, $u v$ is not induced by a bi-set active for $j$.

By the above, $(X, a, j)$ is a reducible triple. Moreover, $u \in V\left(A_{j}\right), v \notin V\left(A_{j}\right)$, so we can extend the $j$ th arborescence by adding $a$ to it.

At the end of the algorithm, we get arborescences $A_{1}, \ldots, A_{t}$ satisfying (3.1) and $A_{i}$ is rooted at $\pi(i)$. We claim that these arborescences give a maximal $\mathcal{M}$ independent packing of arborescences. At the beginning $J_{v}$ is independent for every $v \in V$ by the $\mathcal{M}$-independence of $\pi$, so, by Corollary 3.1, $J_{v} \cup\left\{i: \varrho_{A_{i}}(v) \geq 1\right\}=\{i$ : $\left.v \in V\left(A_{i}\right)\right\}$ is independent. By (3.1),

$$
r\left(\left\{i: v \in V\left(A_{i}\right)\right\}\right)=r\left(J_{v} \cup\left\{i: \varrho_{A_{i}}(v) \geq 1\right\}\right)=r\left(I_{v} \cup J_{v}\right)=r\left(\pi^{-1}\left(\operatorname{Pred}_{v}\right)\right),
$$

as required.

4. Finding a valid arc-cover. Recall that a family of pairwise disjoint arc-sets $A_{1}, \ldots, A_{t}$ of $A$ that satisfies (2.1) is a valid arc-cover. In what follows, we show that a valid arc-cover can be found in polynomial time in an appropriate oracle model.

Let $V^{\prime}$ be a copy of $V$, and identify $\left(X_{I}, X_{O}\right) \in \mathcal{P}_{2}(V)$ with $X_{I} \cup X_{O}^{\prime} \in 2^{V \cup V^{\prime}}$, where $X_{O}^{\prime} \subseteq V^{\prime}$ is the counterpart of $X_{O} \subseteq V$. Note that the union and intersection operations are consistent with this correspondence. Therefore, $\mathcal{F}_{i} \subseteq \mathcal{P}_{2}(V)$ can be regarded as an intersecting family of $2^{V \cup V^{\prime}}$. In what follows in this section, we regard each bi-set in $\mathcal{P}_{2}(V)$ as a subset of $V \cup V^{\prime}$. The definitions of $\mathcal{F}, p$, and active sets are the same as before.

To discuss polynomiality of the algorithm, we need a compact representation of each intersecting family $\mathcal{F}_{i} \subseteq 2^{V \cup V^{\prime}}$. For $v \in V$, let $\mathcal{F}_{i}^{v}:=\left\{X: v \in X \in \mathcal{F}_{i}\right\}$. Since $\mathcal{F}_{i}^{v}$ is closed under the union and intersection operations, it is a ring family (or a distributed lattice), which can be represented by a digraph whose vertex set is a subset of $V \cup V^{\prime}$ by Birkhoff's representation theorem [3] (see also [17]). In what follows, we assume that we are given a digraph representation of $\mathcal{F}_{i}^{v} \subseteq 2^{V \cup V^{\prime}}$ for $v \in V$, and $\mathcal{F}_{i}$ is given as $\bigcup_{v \in V} \mathcal{F}_{i}^{v}$. Note that the size of this representation is polynomial in $|V|$. We also assume the following:

1. $J_{X}$ is given by a membership oracle; i.e., for $X \subseteq V \cup V^{\prime}$ and $i \in[t]$, we can check whether $i \in J_{X}$.

2. The rank function $r$ of the matroid is given as an oracle. That is, for given $S \subseteq[t]$, we can compute $r(S)$.

With these assumptions, we show the following.

Proposition 4.1. In the statement of Theorem 2.2, suppose that $\mathcal{F}_{i}, J_{X}$, and $r$ are given as above, and conditions (AIP) and (IUP) are satisfied. Then, we can check whether condition (2.4) holds or not in polynomial time.

Proof. Let $\mathcal{F}_{+} \subseteq \mathcal{F}$ be the set of all active sets, and for $v \in V$, let $\mathcal{F}_{+}^{v}:=\left\{\bigcup X_{i}\right.$ : $v \in X_{i} \in \mathcal{F}_{+}$for each $\left.i\right\}$.

Claim 4.2. For each $v \in V, \mathcal{F}_{+}^{v}$ is a ring family; i.e., it is closed under the union and intersection operations. Furthermore, the digraph representation of $\mathcal{F}_{+}^{v}$ can be computed in polynomial time.

Proof. It is obvious that $\mathcal{F}_{+}^{v}$ is closed under the union operation. For $X, Y \in \mathcal{F}_{+}^{v}$, suppose that $X=\bigcup X_{i}$ and $Y=\bigcup Y_{j}$, where $v \in X_{i} \in \mathcal{F}_{+}$and $v \in Y_{j} \in \mathcal{F}_{+}$. By (AIP) (or Proposition 2.3), $X_{i} \cap Y_{j} \in \mathcal{F}_{+}$for each $i, j$. Therefore,

$$
X \cap Y=\bigcup_{i, j}\left(X_{i} \cap Y_{j}\right) \in \mathcal{F}_{+}^{v},
$$


which shows that $\mathcal{F}_{+}^{v}$ is closed under the intersection operation.

In order to construct the digraph representation of $\mathcal{F}_{+}^{v}$, it suffices to compute all irreducible elements in $\mathcal{F}_{+}^{v}$, where $X \in \mathcal{F}_{+}^{v}$ is irreducible if there is no pair of sets $Y, Z \in \mathcal{F}_{+}^{v}-X$ such that $X=Y \cup Z$. By the definition of $\mathcal{F}_{+}^{v}$, each irreducible element $X \in \mathcal{F}_{+}^{v}$ is active; i.e., $X \in \mathcal{F}_{i}^{v}$ for some $i \in I_{X}^{a c t}$. Furthermore, $X$ is irreducible in $\mathcal{F}_{i}^{v}$ by the irreducibility in $\mathcal{F}_{+}^{v}$, because if $Y, Z \in \mathcal{F}_{i}^{v}-X$ and $X=Y \cup Z$, then $Y, Z \in \mathcal{F}_{+}^{v}-X$. By this argument,

$$
\left\{X: \exists i \in[t] \text { such that } X \text { is an irreducible element in } \mathcal{F}_{i}^{v} \text { and } i \in I_{X}^{a c t}\right\}
$$

is a subfamily of $\mathcal{F}_{+}^{v}$ containing all irreducible elements. Note that this family can be computed in polynomial time by checking whether each irreducible element in $\mathcal{F}_{i}^{v}$ is active or not for every $i \in[t]$. By using this family, we obtain the digraph representation of $\mathcal{F}_{+}^{v}$.

Claim 4.3. For each $v \in V, p$ is supermodular on the ring family $\mathcal{F}_{+}^{v}$.

Proof. Let $X, Y \in \mathcal{F}_{+}^{v}$. We distinguish three cases:

1. If $p(X)>0$ and $p(Y)>0$, then $p(X)+p(Y) \leq p(X \cap Y)+p(X \cup Y)$ by Proposition 2.4 .

2. If $p(X)=p(Y)=0$, then it is obvious that $p(X)+p(Y)=0 \leq p(X \cap Y)+$ $p(X \cup Y)$.

3. Suppose that $p(X)>0$ and $p(Y)=0$. Since $X \cap Y \in \mathcal{F}_{+}^{v}$ by Claim 4.2, $X \cap Y$ can be represented as $\bigcup Z_{j}$, where $v \in Z_{j} \in \mathcal{F}_{+}$. By (AIP), $Z_{j}$ is active for any $i \in I_{X}^{a c t}$, which implies that $X \cap Y=\bigcup Z_{j} \in \mathcal{F}_{i} \subseteq \mathcal{F}$. Then, by Proposition 2.3 and (IUP), we obtain $p(X) \leq p(X \cap Y)$. By combining this with $p(Y)=0 \leq p(X \cup Y)$, we have $p(X)+p(Y) \leq p(X \cap Y)+p(X \cup Y)$.

This completes the proof.

The digraph $D$ can also be regarded as a bipartite digraph on node set $V \cup V^{\prime}$, where every arc has its head in $V$ and its tail in $V^{\prime}$. It is easy to check that this is consistent with the notion of covering. Condition (2.4) holds if and only if the condition

$$
\varrho(X)-p(X) \geq 0 \text { for every } X \in \mathcal{F}_{+}^{v}
$$

holds for every $v \in V$, and this is equivalent to $\min _{X \in \mathcal{F}_{+}^{v}}\{\varrho(X)-p(X)\} \geq 0$ for every $v \in V$. Since $\varrho-p$ is a submodular function on $\mathcal{F}_{+}^{v}$, we can compute this minimum value in polynomial time by submodular function minimization algorithms (see $[12,17,18])$. Therefore, condition (2.4) can be checked in polynomial time.

Corollary 4.4. In the statement of Theorem 2.2, suppose that $\mathcal{F}_{i}, J_{X}$, and $r$ are given as above, and conditions (AIP), (IUP), and (2.4) are satisfied. Then we can find a valid arc-cover in polynomial time.

Proof. For any arc $a=u v \in A$ and for any index $i \in[t]$, we can check using the digraph representation of $\mathcal{F}_{i}^{v}$ whether the unique smallest bi-set in $\mathcal{F}_{i}$ that induces $a$ is active for $i$. If it is not, then we try the following procedure:

- adding $a$ to $A_{i}$ and removing it from $A$;

- adding $i$ to $J_{X}$ for every bi-set $X \in \mathcal{F}_{i}$ covered by $a$; and

- removing bi-sets covered by $a$ from $\mathcal{F}_{i}$.

The new instance obtained by this procedure still satisfies (AIP), because $a$ is not induced by any bi-set active for $i$. The new digraph representations for $\mathcal{F}_{i}$ and the oracle for $J_{X}$ are easy to construct using the original ones. 
It is possible that the new instance does not satisfy condition (2.4). However, we know that there exists a pair $(a, i)$ such that condition (2.4) still holds after this procedure, and by Proposition 4.1 we can check whether it holds in polynomial time. Thus we can check all pairs $(a, i)$ and choose one where condition (2.4) is satisfied in the new instance. By repeating this procedure, $A$ becomes empty and the obtained arc sets $A_{1}, \ldots A_{t}$ form a valid arc-cover.

5. Finding a minimum cost arc-cover. The main tool used in this section is the following theorem of Frank [7]; see also [8, Theorem 17.1.11].

Theorem 5.1 (Frank). Let $D=(V, A)$ be a digraph, $q$ be a positively intersecting supermodular bi-set function on $V$, and $g \in \mathbb{Z}_{+}^{A}$ be a nonnegative upper bound on the arcs. Then the system

$$
\left\{x \in \mathbb{R}^{A}: 0 \leq x_{a} \leq g_{a} \forall a \in A, \varrho_{x}(Z) \geq q(Z) \forall Z \in \mathcal{P}_{2}(V)\right\}
$$

is total dual integral, where $\varrho_{x}(Z)=\sum_{a \in \Delta^{i n}(Z)} x_{a}$. If $q$ is the nonnegative part of an intersecting supermodular bi-set function, then the system defines a submodular flow polyhedron.

We first describe a polynomial algorithm that uses the ellipsoid method.

Theorem 5.2. Let $D=(V, A)$ be a digraph, let $c: A \rightarrow \mathbb{R}$ be a cost function, and suppose that $\mathcal{F}_{i}, J_{X}$, and $r$ are given as in section 4 , so that (AIP) and (IUP) are satisfied. We can find a minimum cost arc set $A^{\prime} \subseteq A$ that satisfies

$$
\varrho_{A^{\prime}}(X) \geq p(X) \quad \text { for every } X \in \mathcal{F}
$$

in polynomial time.

Proof. Let

$$
P=\left\{x \in \mathbb{R}^{A}: 0 \leq x_{a} \leq 1 \forall a \in A, \varrho_{x}(Z) \geq p(Z) \forall Z \in \mathcal{F}\right\} .
$$

The set function $p$ is positively intersecting supermodular by Proposition 2.4, so by Theorem 5.1 the system of inequalities defining $P$ is total dual integral. As a consequence, the polyhedron $P$ is integer, so finding a minimum cost valid arc-cover amounts to optimization over $P$. By Proposition 4.1, there is a polynomial-time separation algorithm for the linear system defining $P$, and therefore we can optimize on $P$ in polynomial time using the ellipsoid method by the results of Grötschel, Lovász, and Schrijver [11].

It is left as an open question whether there is a strongly polynomial combinatorial algorithm for finding the minimum cost valid arc-cover. However, in the special case when $\mathcal{F}$ is an intersecting family, this is possible using submodular flows.

Theorem 5.3. Let $D=(V, A)$ be a digraph, and let $c: A \rightarrow \mathbb{R}$ be a cost function. Let $\mathcal{F}$ be an intersecting family over $\mathcal{P}_{2}$, and suppose that $\mathcal{F}_{i}, J_{X}$, and $r$ are given as in section 4 , so that (AIP) and (IUP) are satisfied, and each $\mathcal{F}_{i}$ is a subfamily of $\mathcal{F}$. Then we can find a minimum cost arc set $A^{\prime} \subseteq A$ that satisfies

$$
\varrho_{A^{\prime}}(X) \geq p(X) \quad \text { for every } X \in \mathcal{F}
$$

in strongly polynomial time. 
Proof. Let

$$
p^{\prime}(X)= \begin{cases}r\left(I_{X} \cup J_{X}\right)-r\left(J_{X}\right) & \text { if } X \in \mathcal{F}, \\ -\infty & \text { otherwise. }\end{cases}
$$

The main advantage of $\mathcal{F}$ being intersecting is that it implies the intersecting supermodularity of $p^{\prime}$.

Proposition 5.4. The bi-set function $p^{\prime}$ is intersecting supermodular on $\mathcal{P}_{2}(V)$.

Proof. Let $X$ and $Y$ be intersecting bi-sets. If $p^{\prime}(X)$ or $p^{\prime}(Y)$ is $-\infty$, then the supermodular inequality trivially holds.

If $p^{\prime}(X), p^{\prime}(Y) \geq 0$, then $X, Y \in \mathcal{F}$. As $\mathcal{F}$ is intersecting, $X \cap Y$ and $X \cup Y$ are also in $\mathcal{F}$. Note that Proposition 2.3 implies $J_{X \cap Y} \subseteq \operatorname{Span}\left(J_{X}\right) \cap \operatorname{Span}\left(J_{Y}\right)$. As the families $\mathcal{F}_{i}$ are also intersecting, we have $I_{X \cup Y} \supseteq I_{X} \cap I_{Y}$. This, together with (IUP), implies $J_{X \cup Y} \subseteq J_{X} \cup J_{Y}$. Thus

$$
\begin{aligned}
r\left(J_{X}\right)+r\left(J_{Y}\right) & =r\left(\operatorname{Span}\left(J_{X}\right)\right)+r\left(\operatorname{Span}\left(J_{Y}\right)\right) \\
& \geq r\left(\operatorname{Span}\left(J_{X}\right) \cap \operatorname{Span}\left(J_{Y}\right)\right)+r\left(\operatorname{Span}\left(J_{X}\right) \cup \operatorname{Span}\left(J_{Y}\right)\right) \\
& \geq r\left(J_{X \cap Y}\right)+r\left(J_{X \cup Y}\right) .
\end{aligned}
$$

Using this and (IUP), we get

$$
\begin{aligned}
p^{\prime}(X)+p^{\prime}(Y) & =r\left(I_{X} \cup J_{X}\right)-r\left(J_{X}\right)+r\left(I_{Y} \cup J_{Y}\right)-r\left(J_{Y}\right) \\
& \leq r\left(I_{X \cap Y} \cup J_{X \cap Y}\right)-r\left(J_{X \cap Y}\right)+r\left(I_{X \cup Y} \cup J_{X \cup Y}\right)-r\left(J_{X \cup Y}\right) \\
& =p^{\prime}(X \cap Y)+p^{\prime}(X \cup Y) .
\end{aligned}
$$

As $p$ is the nonnegative part of $p^{\prime}$, Theorem 5.1 of Frank implies that the system

$$
\left\{x \in \mathbb{R}^{A}: 0 \leq x_{a} \leq 1 \forall a \in A, \varrho_{x}(Z) \geq p(Z) \forall Z \in \mathcal{P}_{2}(V)\right\}
$$

describes a submodular flow polyhedron. Moreover, the proof of Frank gives a construction of a corresponding submodular flow problem that in our case can be solved in strongly polynomial time, since the support of $p^{\prime}$ is given by the digraph representations.

6. Open questions. Let $D=\left(V+r_{0}, A \cup S\right)$ be a directed graph, where $S=$ $\left\{a_{1}, \ldots, a_{t}\right\}=\Delta^{\text {out }}\left(r_{0}\right)$. For an $r_{0}$-arborescence $F \subseteq A \cup S$ and node $v \in V$, the unique $r_{0}-v$ path in $F$ is denoted by $F\left(r_{0}, v\right)$, and the index of the first arc of $F\left(r_{0}, v\right)$ is denoted by $i_{v}^{F}$. In other words, $i_{v}^{F}=j$ if and only if $F\left(r_{0}, v\right) \cap S=a_{j}$. For a subset $X \subseteq V$, let $P_{X}=\left\{i\right.$ : there is a path from $r_{0}$ to $X$ through $\left.a_{i}\right\}$, and let $\Delta_{S}^{i n}(X)=\Delta^{i n}(X) \cap S$.

The main result of [4] can be reformulated as follows.

Theorem 6.1. Let $D=\left(V+r_{0}, A \cup S\right)$ be a digraph, where $S=\left\{a_{1}, \ldots, a_{t}\right\}=$ $\Delta^{\text {out }}\left(r_{0}\right)$. Let $\mathcal{M}=([t], r)$ be a matroid of rank $k$. There exist $t$ pairwise arc-disjoint $r_{0}$-arborescences $F_{1}, \ldots, F_{t}$ such that $a_{i} \in F_{i}$ for $i=1, \ldots, t$ and $\left\{i: v \in V\left(F_{i}\right)\right\}$ forms a base of $\mathcal{M}$ for each $v \in V$ if and only if $\Delta_{S}^{i n}(v)$ is independent for every $v \in V$ and

$$
\varrho_{A}(X) \geq k-r\left(\Delta_{S}^{i n}(X)\right) \quad \text { for every } \emptyset \neq X \subseteq V .
$$

However, a direct extension of Edmonds's theorem would be the following. 
Conjecture 6.2. Let $D=\left(V+r_{0}, A \cup S\right)$ be a digraph, where $S=\left\{a_{1}, \ldots, a_{t}\right\}=$ $\Delta^{\text {out }}\left(r_{0}\right)$. Let $\mathcal{M}=([t], r)$ be a matroid of rank $k$. There exist $k$ pairwise arc-disjoint spanning $r_{0}$-arborescences $F_{1}, \ldots, F_{k}$ such that $\left\{i_{v}^{F_{j}}: j=1, \ldots, k\right\}$ forms a base of $\mathcal{M}$ for each $v \in V$ if and only if

$$
\varrho_{A}(X) \geq k-r\left(\Delta_{S}^{i n}(X)\right) \quad \text { for every } \emptyset \neq X \subseteq V .
$$

It can be verified that, if true, Conjecture 6.2 would imply Theorem 6.1. Also, it is worth mentioning the following analogy. In the case of Edmonds's theorem, (1.1) is equivalent to the rooted $k$-arc-connectivity of $D$, that is, when there are $k$ pairwise arc-disjoint directed paths from $r_{0}$ to $v$ for each $v \in V-r_{0}$. In Conjecture 6.2, (6.1) is equivalent to a matroidal version of rooted connectivity. We call a set of directed $r_{0}-v$ paths $\mathcal{M}$-independent if they are pairwise arc-disjoint and the first arcs of these paths form an independent set of $\mathcal{M}$. It can be shown that (6.1) is equivalent to the existence of $k \mathcal{M}$-independent $r_{0}-v$ paths for every $v \in V$.

A similar reformulation of Theorem 1.2 is as follows.

Theorem 6.3. Let $D=\left(V+r_{0}, A \cup S\right)$ be a digraph, where $S=\left\{a_{1}, \ldots, a_{t}\right\}=$ $\Delta^{\text {out }}\left(r_{0}\right)$. Let $\mathcal{M}=([t], r)$ be a matroid. There exist $t$ pairwise arc-disjoint $r_{0}$ arborescences $F_{1}, \ldots, F_{t}$ such that $a_{i} \in F_{i}$ for $i=1, \ldots, t,\left\{i: v \in V\left(F_{i}\right)\right\}$ is independent in $\mathcal{M}$, and $r\left(\left\{i: v \in V\left(F_{i}\right)\right\}\right)=r\left(P_{v}\right)$ for each $v \in V$ if and only if $\Delta_{S}^{\text {in }}(v)$ is independent for every $v \in V$ and

$$
\varrho_{A}(X) \geq r\left(P_{X}\right)-r\left(\Delta_{S}^{i n}(X)\right) \quad \text { for every } \emptyset \neq X \subseteq V .
$$

A strengthening of Conjecture 6.2 would be the following.

Conjecture 6.4. Let $D=\left(V+r_{0}, A\right)$ be a digraph, where $S=\left\{a_{1}, \ldots, a_{t}\right\}=$ $\Delta^{\text {out }}\left(r_{0}\right)$. Let $\mathcal{M}=([t], r)$ be a matroid of rank $k$. There exist $k$ pairwise arc-disjoint $r_{0}$-arborescences $F_{1}, \ldots, F_{k}$ such that $\left\{i_{v}^{F_{j}}: v \in V\left(F_{j}\right)\right\}$ is independent in $\mathcal{M}$ and $r\left(\left\{i_{v}^{F_{j}}: v \in V\left(F_{j}\right)\right\}\right)=r\left(P_{v}\right)$ for each $v \in V$ if and only if

$$
\varrho_{A}(X) \geq r\left(P_{X}\right)-r\left(\Delta_{S}^{i n}(X)\right) \quad \text { for every } \emptyset \neq X \subseteq V .
$$

Conjectures 6.2 and 6.4 basically state that the $t$ arborescences appearing in Theorems 6.1 and 6.3 can be chosen in a very special way: they can be divided into $k$ groups such that the node-sets of arborescences corresponding to the same group are disjoint apart from the root-node. Besides the validity of the above conjectures, their connection to Theorem 2.2 is also of interest.

Another possible strengthening of these theorems would be to put some restrictions on the node-sets spanned by the arborescences in question. A natural candidate for a restriction is convexity, introduced in [9]. Given a digraph $D=(V, A)$, a subset $U$ of nodes is convex if there is no node $v \in V-U$ such that $v$ is reachable from $U$ and $U$ is reachable from $v$. This would suggest the following variant of Theorem 6.3.

Conjecture 6.5. Let $D=\left(V+r_{0}, A \cup S\right)$ be a digraph, where $S=\left\{a_{1}, \ldots, a_{t}\right\}=$ $\Delta^{\text {out }}\left(r_{0}\right)$, and let $\mathcal{M}=([t], r)$ be a matroid. There exist $t$ pairwise arc-disjoint $r_{0}$ arborescences $F_{1}, \ldots, F_{t}$ such that $V\left(F_{i}\right)$ is a convex set and $a_{i} \in F_{i}$ for $i=1, \ldots, t$, $\left\{i: v \in V\left(F_{i}\right)\right\}$ is independent in $\mathcal{M}$, and $r\left(\left\{i: v \in V\left(F_{i}\right)\right\}\right)=r\left(P_{v}\right)$ for each $v \in V$ if and only if $\Delta_{S}^{i n}(v)$ is independent for every $v \in V$ and

$$
\varrho_{A}(X) \geq r\left(P_{X}\right)-r\left(\Delta_{S}^{i n}(X)\right) \quad \text { for every } \emptyset \neq X \subseteq V .
$$


However, it turns out that Conjecture 6.5 is false. A counterexample can be constructed as follows. Let $V=\left\{v_{1}, v_{2}, v_{3}\right\}, S=\left\{r_{0} v_{1}, r_{0} v_{2}, r_{0} v_{3}\right\}$, and $A=\left\{v_{1} v_{2}\right.$, $\left.v_{2} v_{3}, v_{3} v_{1}\right\}$. Let $\mathcal{M}=(S, r)$ be the uniform matroid on $S$ of rank 2 . It is not difficult to see that $D$ and $r$ satisfy (6.2), but there is no solution in which each arborescence spans a convex set.

Finally, we show that a further extension of Conjecture 6.4 to root-sets results in an NP-complete problem. We call a collection of node-disjoint arborescences a branching. Equivalently, a branching $(U, B)$ is a directed forest in which each node has in-degree at most one. The set of nodes having in-degree zero is called the root-set of the branching. For a digraph $D=(V, A)$ and $\emptyset \neq R \subseteq V$, a branching $(V, B)$ is called a spanning $R$-branching if its root-set is exactly $R$.

Let $D=(V, A)$ be a digraph, and let $\mathcal{R}=\left\{R_{1}, \ldots, R_{k}\right\}$ be a list of root-sets. Let $t=\sum\left|R_{i}\right|$, let $\mathcal{M}=([t], r)$ be a matroid of rank $k$, and let $f$ be a bijection from the multiset $R_{1}+\cdots+R_{k}$ to [t]. If $B$ is a branching, $v$ is in $V(B)$, and $r_{0}$ is the root of the arborescence in $B$ that contains $v$, then we use the notation $i_{v}^{B}=f\left(r_{0}\right)$. Let $P_{v}=\left\{f(u): u \in R_{1}+\cdots+R_{k}, v\right.$ is reachable from $\left.u\right\}$.

Problem 6.6. Can we decide in polynomial time the existence of $k$ pairwise arcdisjoint branchings $B_{1}, \ldots, B_{k}$ such that $B_{i}$ is rooted at $R_{i},\left\{i_{v}^{B_{j}}: v \in V\left(B_{j}\right)\right\}$ is independent in $\mathcal{M}$, and $r\left(\left\{i_{v}^{B_{j}}: v \in V\left(B_{j}\right)\right\}\right)=r\left(P_{v}\right)$ for each $v \in V$ ?

Theorem 6.7. Problem 6.6 is NP-complete, even with the restriction that $\mathcal{M}$ is a partition matroid.

Proof. Let $R$ be a ground set and $\mathcal{M}_{1}, \mathcal{M}_{2}$, and $\mathcal{M}_{3}$ be partition matroids on $R$ with $r_{i}(R)=k$ for $i=1,2,3$. Deciding the existence of a common base of three partition matroids in general is NP-complete, as the three-dimensional matching problem [10, Problem SP1] can be reduced to it easily.

Let $R_{1}, \ldots, R_{k}$ denote the partition classes of $\mathcal{M}_{1}$; that is, a set $X \subseteq R$ is independent in $\mathcal{M}_{1}$ if and only if $\left|X \cap R_{i}\right| \leq 1$ for each $i=1, \ldots, k$. As partition matroids are special cases of gammoids, there is a digraph $D=(R+T, A)$ such that $R$ is a stable set with $\varrho(R)=0$ and a set $X \subseteq R$ is independent in $\mathcal{M}_{2}$ if and only if there exist $|X|$ directed paths - node-disjoint apart from $v_{0}$-from $X$ to a fixed node $v_{0} \in T$.

Note that $\mathcal{M}_{3}$ is also defined in $R$; hence let $f$ be the identity function. For each $v \in T-v_{0}$, add $k$ directed arcs going from $v_{0}$ to $v$ to the digraph. Then the existence of $k$ pairwise arc-disjoint branchings $B_{1}, \ldots, B_{k}$ satisfying the conditions of Problem 6.6 with the above $f$ and $\mathcal{M}=\mathcal{M}_{3}$ is equivalent to the existence of a common base of the $\mathcal{M}_{i}$ 's.

Acknowledgment. The work was partially done while the first and second authors visited the Department of Mathematical Informatics, University of Tokyo, Tokyo, Japan, 2013.

\section{REFERENCES}

[1] J. Bang-Jensen And G. Gutin, Digraphs: Theory, Algorithms and Applications, 2nd ed., Springer-Verlag, London, 2008.

[2] K. BÉrCZi And A. Frank, Variations for Lovász' submodular ideas, in Building Bridges, Springer, Berlin, 2008, pp. 137-164, doi:10.1007/978-3-540-85221-6_4.

[3] G. Birkhoff, Lattice Theory, Amer. Math. Soc. Colloq. Publ. 25. American Mathematical Society, Providence, RI, 1967. 
[4] O. Durand de Gevigney, V.-H. Nguyen, and Z. Szigeti, Matroid-based packing of arborescences, SIAM J. Discrete Math., 27 (2013), pp. 567-574, doi:10.1137/120883761.

[5] J. Edmonds, Edge-disjoint branchings, in Combinatorial Algorithms, Courant Comput. Sci. Sympos. 9, Algorithmics Press, New York, 1973, pp. 91-96.

[6] A. Frank, Kernel systems of directed graphs, Acta Sci. Math. (Szeged), 41 (1979), pp. 63-76.

[7] A. Frank, Rooted k-connections in digraphs, Discrete Appl. Math., 157 (2009), pp. 1242-1254, doi:10.1016/j.dam.2008.03.040.

[8] A. Frank, Connections in Combinatorial Optimization, Oxford Lecture Ser. Math. Appl. 38, Oxford University Press, Oxford, UK, 2011.

[9] S. Fujishige, A note on disjoint arborescences, Combinatorica, 30 (2010), pp. 247-252, doi:10. 1007/s00493-010-2518-y.

[10] M. R. Garey And D. S. Johnson, A Guide to the Theory of NP-Completeness, W.H. Freeman, New York, 1979.

[11] M. Grötschel, L. Lovász, And A. Schrijver, The ellipsoid method and its consequences in combinatorial optimization, Combinatorica, 1 (1981), pp. 169-197, doi:10.1007/ BF02579273.

[12] S. Iwata, L. Fleischer, and S. Fujishige, A combinatorial strongly polynomial algorithm for minimizing submodular functions, J. ACM, 48 (2001), pp. 761-777, doi:10.1145/502090. 502096.

[13] N. Kamiyama, N. Katoh, And A. Takizawa, Arc-disjoint in-trees in directed graphs, Combinatorica, 29 (2009), pp. 197-214; available from http://link.springer.com/article/10.1007/ s00493-009-2428-z.

[14] N. Katoh and S.-I. Tanigawa, Rooted-tree decompositions with matroid constraints and the infinitesimal rigidity of frameworks with boundaries, SIAM J. Discrete Math., 27 (2013), pp. 155-185, doi:10.1137/110846944.

[15] Cs. KIRÁly, On Maximal Independent Arborescence-Packing, Technical Report TR-2013-03, Egerváry Research Group, Eötvös University, Budapest, Hungary, 2013.

[16] L. LovÁsz, On two minimax theorems in graph, J. Combinatorial Theory Ser. B, 21 (1976), pp. 96-103, doi:10.1016/0095-8956(76)90049-6.

[17] S. T. MCCormick, Submodular function minimization, in Handbooks in Operations Research and Management Science, Elsevier, Amsterdam, 2006, pp. 321-391, doi:10.1016/ S0927-0507(05)12007-6.

[18] A. SchriJver, A combinatorial algorithm minimizing submodular functions in strongly polynomial time, J. Combin. Theory Ser. B, 80 (2000), pp. 346-355, doi:10.1006/jctb.2000. 1989.

[19] L. SzeGŐ, On covering intersecting set-systems by digraphs, Discrete Math., 234 (2001), pp. 187-189, doi:10.1016/S0012-365X(00)00381-2. 